\title{
Impact of Immunotherapy on the Survival of Patients With Cancer and Brain Metastases
}

\author{
Saber Amin, $\mathrm{MD}^{1}$; Michael Baine, MD, $\mathrm{PhD}^{1}$; Jane Meza, $\mathrm{PhD}^{2}$; and Chi Lin, MD, $\mathrm{PhD}^{1}$
}

\section{ABSTRACT}

Background: Immunotherapy has shown excellent efficacy in various cancers. However, there is a lack of knowledge regarding the significant role of immunotherapy in patients with brain metastases (BMs). The objective of this study was to investigate, using the $\mathrm{Na}$ tional Cancer Database, the impact of immunotherapy on the overall survival (OS) of patients with BMs who did not receive definitive surgery of the primary tumor. Patients and Methods: Patients diagnosed with the primary cancer of non-small cell lung cancer, small cell lung cancer, other types of lung cancer, breast cancer, melanoma, colorectal cancer, or renal cancer who had BMs at the time of diagnosis were identified from the National Cancer Database. We assessed OS using a Cox proportional hazards model adjusted for age at diagnosis, sex, race, education level, income level, residential area, treatment facility type, insurance status, Charlson-Deyo comorbidity status, year of diagnosis, primary tumor type, and receipt of chemotherapy, radiation therapy (RT), and/or immunotherapy, because these factors were significantly associated with OS in the univariable analysis. Results: Of 94,215 patients who were analyzed, 3,097 (3.29\%) received immunotherapy. In the multivariable analysis, immunotherapy was associated with significantly improved OS (hazard ratio [HR], 0.694; 95\% Cl, 0.664-0.726; $P<.0001$ ) compared with no immunotherapy. Treatment using chemotherapy plus immunotherapy was significantly associated with improved OS (HR, $0.643 ; 95 \% \mathrm{Cl}, 0.560-0.738 ; P<.0001)$ compared with chemotherapy without immunotherapy. RT plus immunotherapy was also associated with significantly improved OS $(\mathrm{HR}, 0.389 ; 95 \% \mathrm{Cl}, 0.352-0.429$; $P<.0001)$ compared with RT alone. Furthermore, chemoradiation (CRT) plus immunotherapy was associated with significantly improved OS (HR, 0.793; 95\% Cl, 0.752-0.836; $P<.0001)$ compared with CRT alone. Conclusions: In this comprehensive analysis, the addition of immunotherapy to chemotherapy, RT, and CRT was associated with significantly improved OS in patients with BMs. The study warrants future clinical trials of immunotherapy in patients with BMs, who have historically been excluded from these trials.

J Natl Compr Canc Netw 2020;18(7):832-840 doi: $10.6004 /$ jnccn.2020.7547

${ }^{1}$ Department of Radiation Oncology, College of Medicine, and ${ }^{2}$ Department of Biostatistics, College of Public Health, University of Nebraska Medical Center, Omaha, Nebraska.

\section{Background}

Brain metastases (BMs) are the most common intracranial malignancy, occurring in $20 \%$ to $40 \%$ of all patients with cancer. $^{1}$ Each year, $>150,000$ people are diagnosed with BMs in the United States. ${ }^{2}$ The incidence rate of BMs is estimated to be between 8.3 and 14.3 per 100,000 population. ${ }^{1}$ The actual incidence rate may be higher because most of the studies reporting the incidence of BMs predate modern imaging and treatments. ${ }^{1}$ Furthermore, improved imaging and prolonged survival of patients with primary cancer attributed to advances in cancer therapeutics and screening have led to an increased incidence of BMs. ${ }^{3}$ Despite improvements in radiation and systemic treatments, the overall survival (OS) of patients with BMs remains dismal, with a median survival time of 4 to 16 months, depending on the primary site..$^{4-6}$

Current treatment modalities available for the treatment of BMs are surgery, whole-brain radiation therapy (RT), and stereotactic radiosurgery (SRS). ${ }^{7,8}$ The most appropriate treatment option is selected based on BM size, number, histology, and location; primary tumor type; control of systemic disease; symptoms; past cancer diagnosis; and patient performance status. ${ }^{9}$ whole-brain RT is typically used for multiple brain lesions, surgery is reserved for a more significant or symptomatic lesion, and SRS is used for lesions $\leq 4 \mathrm{~cm}$ to improve local control. ${ }^{10}$ Surgery is rarely used without adjuvant wholebrain RT or SRS, due to higher recurrence. The utility of systemic therapy in the treatment of BMs remains somewhat unclear because many drugs that are highly efficacious in the treatment of extracranial cancers show poor efficacy within the brain. With the progress in understanding of the $\mathrm{BM}$ microenvironment and the pathophysiology of the blood-brain barrier (BBB), as well as the development of cytotoxic agents and targeted therapies, a renewed interest has been shown in the use of systemic treatment for BMs. ${ }^{11,12}$ Currently, there is a

\section{See JNCCN.org for supplemental online content.}


compelling rationale that systemic therapies that sufficiently penetrate the BBB can evoke a clinical response in the central nervous system. ${ }^{13}$

Increasing evidence shows that some targeted therapies may treat and prevent BMs. ${ }^{9,11}$ Some studies have shown significant intracranial efficacy of HER2 inhibitors, epidermal growth factor receptor inhibitors, BRAF kinase inhibitors, and tyrosine kinase inhibitors in BMs from breast, non-small cell lung cancer (NSCLC), melanoma, and renal cell carcinoma and thus could be used in individual patients. ${ }^{14-17}$ An HER2 inhibitor improved median survival by approximately 15 months in patients with BMs from breast cancer (28.6 vs 12.9 months).$^{18}$ An epidermal growth factor receptor inhibitor improved median OS by 3 months in patients with BMs from NSCLC (12 months in targeted therapy vs 9 months in chemotherapy). ${ }^{19}$ Median survival was improved by 16 months in patients with BMs from melanoma receiving a BRAF kinase inhibitor (21.3 vs 4.9 months). ${ }^{20}$ A tyrosine kinase inhibitor improved median survival in patients with BMs from renal cell carcinoma by more than 9 months (16.6 vs 7.2 months). ${ }^{21}$

The development of immunotherapeutics offers an exciting opportunity for the treatment of BMs. ${ }^{12}$ Immunotherapy has shown great success in the treatment of various tumors, but the main question in BMs is whether immunotherapy antibodies can cross the BBB. ${ }^{11,22}$ The brain was long considered an immuneprivileged organ, and it was thought that immunotherapy was ineffective in BMs because it will not cross the BBB or that even when it crossed the BBB, its ability to elicit a robust immune response would be limited. ${ }^{11,12}$ However, increasing evidence suggests that $\mathrm{T}$ cells and tumor-infiltrating lymphocytes traffic to the brain, and their presence is associated with improved survival and better response to immunotherapy. ${ }^{23-25}$

Clinical evidence, although limited, shows that monoclonal antibodies can cross the BBB and thus could be another potential option for the treatment of BMs. ${ }^{26}$ Preclinical studies indicate that immunotherapy can penetrate the $\mathrm{BBB}$ in both primary and metastatic brain cancer. ${ }^{7} \mathrm{~A}$ few retrospective studies have investigated the impact of immunotherapy on the survival of BMs, but almost all of them included only BMs from melanoma and focused on just a single agent, ipilimumab. ${ }^{27-29}$ More extensive studies investigating the impact of immunotherapy on the survival of BMs are lacking, and there is a lack of understanding about the potential role of immunotherapy in BMs, regardless of the primary tumor. Our study includes only patients who did not undergo surgery of the primary site, because their survival is different from those who do undergo surgery (9 vs 20 months) based on our data. We assume these 2 groups are different patient populations.

\section{Patients and Methods}

\section{Data Source}

Data for our study were extracted from the National Cancer Database (NCDB), which is a joint program of the Commission on Cancer of the American College of Surgeons and the American Cancer Society. The NCDB captures $\geq 70 \%$ of newly diagnosed malignancies in the United States annually. Because all patient information is deidentified, this study was exempt from Institutional Review Board evaluation.

\section{Study Population}

Patients aged $\geq 18$ years who were diagnosed with NSCLC, small cell lung cancer (SCLC), other types of lung cancer, breast cancer, colorectal cancer (CRC), melanoma, or renal cell carcinoma between 2010 and 2015 and who had distant metastatic involvement of the brain (regardless of the number of metastases and size of the tumor) at the time of diagnosis were included in this study. Patients who were diagnosed in 2016 were excluded because they did not have a minimum follow-up of 1 year. 2010 was the first year that the NCDB began collecting information about the presence of distant metastatic involvement of the brain at the time of diagnosis of primary cancer. Patients who received definitive surgery of the primary site and those who were missing information about RT, chemotherapy, and immunotherapy were excluded from the analysis. Patients with unknown or missing information about other covariates were not included in the adjusted multivariable analysis.

\section{Endpoints}

The primary outcome was OS, which was calculated from the date of diagnosis to the date of death. Those alive or lost to follow-up were censored. We also reported the predictors of receiving immunotherapy by reporting the odds ratio (OR).

\section{Predictors and Covariates}

The main predictors were immunotherapy, immunotherapy combined with RT, immunotherapy combined with chemotherapy, and immunotherapy combined with chemoradiation (CRT). Other covariates included age at diagnosis, sex, race, education level, income level, residential area, treatment facility type, insurance status, Charlson-Deyo comorbidity score, year of diagnosis, primary tumor type, and receipt of chemotherapy, surgery, RT, and immunotherapy.

\section{Statistical Analyses}

Descriptive statistics for categorical and continuous variables are reported. Predictors of immunotherapy use were identified using the logistic regression model. 
The OR was reported as the measure of association with likelihood of using immunotherapy. Survival time was measured in months from the date of diagnosis to the date of death. We used the Kaplan-Meier (KM) method to generate survival curves and analyzed the differences between groups using the log-rank test.

Cox proportional hazards regression analysis was conducted to estimate the hazard ratio (HR) and its associated 95\% CI. The multivariable Cox regression model included variables significant at $P<.20$ in univariable models. We used the $P$ value of .05 to define statistical significance. We developed separate multivariable models for reporting the HR of immunotherapy combined with other standard treatments, because these combinations are mutually exclusive variables. We used SAS 9.4 (SAS Institute Inc.) for the analysis.

\section{Results}

A total of 97,399 patients who were diagnosed between 2010 and 2015 met the inclusion criteria and were identified from the NCDB. We excluded 3,184 patients because they had missing information about chemotherapy, RT, and immunotherapy. The final sample size was 94,215, of whom 62,255 (66.08\%) had NSCLC, 15,271 (16.21\%) had SCLC, 6,526 (6.93\%) had other type of lung cancer, 3,783 (4.02\%) had breast cancer, 3,291 (3.49\%) had melanoma, 2,198 (2.33\%) had renal cell carcinoma, and $891(0.95 \%)$ had CRC. In terms of immunotherapy use, 3,097 (3.29\%) had received immunotherapy and 91,118 (96.71\%) did not.

Median age of the study population was 65 years, with a range of 19 to 90 years. Most patients were white, aged $>50$ years, and living in urban areas. Older age; receipt of chemotherapy or RT; tumor type of breast, NSCLC, melanoma, and CRC; lower Charlson-Deyo comorbidity score; treatment in an academic facility; and recent year of diagnosis were positively associated with use of immunotherapy on multivariable analysis. The ORs of receiving immunotherapy for the variables of interest are provided in Table 1.

KM curves showed improved survival for patients who received immunotherapy compared with those who did not (Figure 1A). Significantly improved OS was also noted when immunotherapy was combined with other standard-of-care treatment regimens. Chemotherapy + immunotherapy was associated with better OS than chemotherapy alone; RT + immunotherapy had better OS than RT alone; and CRT + immunotherapy had better OS than CRT alone (Figure 1B-1D). Patients who received immunotherapy had an absolute median OS benefit of 5.65 months (14.65 vs 9.00 months) compared with their counterparts who did not receive immunotherapy. An absolute median OS benefit of 3.5 months was noted for chemotherapy + immunotherapy versus chemotherapy alone; 9.11 months for RT + immunotherapy versus RT alone; and 1.9 months for CRT + immunotherapy versus CRT alone.

We also performed KM analysis for the combination of immunotherapy with standard-of-care treatments separately for each of the primary tumors of the breast, NSCLC, and melanoma to check whether the effect of these combination treatments was different for different histologies. Our sample size was sufficient only for these 3 cancers. Results were the same as reported in the overall analysis of this study, except that in patients with melanoma, only RT + immunotherapy showed a survival benefit compared with RT alone. Supplemental eFigures 1-3 show the details of KM analysis by primary disease.

In the univariable Cox proportional hazards analysis (Table 2), immunotherapy was associated with significantly improved OS, with an HR of 0.521 (95\% CI, $0.500-0.543 ; P<.001)$. Significantly improved OS was also noted in immunotherapy + chemotherapy versus chemotherapy alone (HR, 0.580; 95\% CI, 0.507-0.664; $P<.001$ ) (Table 3 ), immunotherapy + RT versus RT alone (HR, 0.361; 95\% CI, 0.328-0.396; $P<.001$ ), and immunotherapy + CRT versus CRT alone (HR, 0.724; 95\% CI, $0.688-0.762 ; P<.001$ ). Receipt of immunotherapy; female sex; black and nonwhite/nonblack race; and tumor type of breast, NSCLC, SCLC, and CRC were associated with improved OS. Advanced age, low education level, low income level, living in rural areas, treatment in a community facility, Charlson-Deyo comorbidity score of 1 or 2 , year of diagnosis between 2010 and 2013, tumor type of other lung cancer, and not receiving chemotherapy or RT were associated with decreased OS in the univariable analysis.

In the multivariable Cox proportional hazards analysis (Table 2), receipt of immunotherapy, chemotherapy, RT, or surgery; female sex; black or nonwhite/nonblack race; and tumor type of breast and CRC were associated with improved OS. However, treatment in a community facility; Charlson-Deyo comorbidity score of 1 or 2; no insurance; year of diagnosis between 2010 and 2013; tumor type of SCLC, other types of lung cancer, and melanoma; and not receiving chemotherapy or RT were associated with decreased OS. In the multivariable analysis adjusted for all of these factors, immunotherapy was associated with significantly improved OS (HR, 0.694; 95\% CI, 0.664-0.726; $P<.0001$ ) compared with no immunotherapy. Treatment with chemotherapy + immunotherapy was significantly associated with improved OS (HR, 0.643; 95\% CI, 0.560-0.738; $P<.0001$ ) (Table 2) compared with chemotherapy without immunotherapy. RT + immunotherapy was also associated with significantly improved OS (HR, 0.389; 95\% CI, 0.352-0.429; $P<.0001$ ) (Table 3) compared with RT alone. Furthermore, CRT + immunotherapy was associated with significantly 


\section{Table 1. Multivariable Logistic Regression Analysis of Factors Associated With Receipt of Immunotherapy}

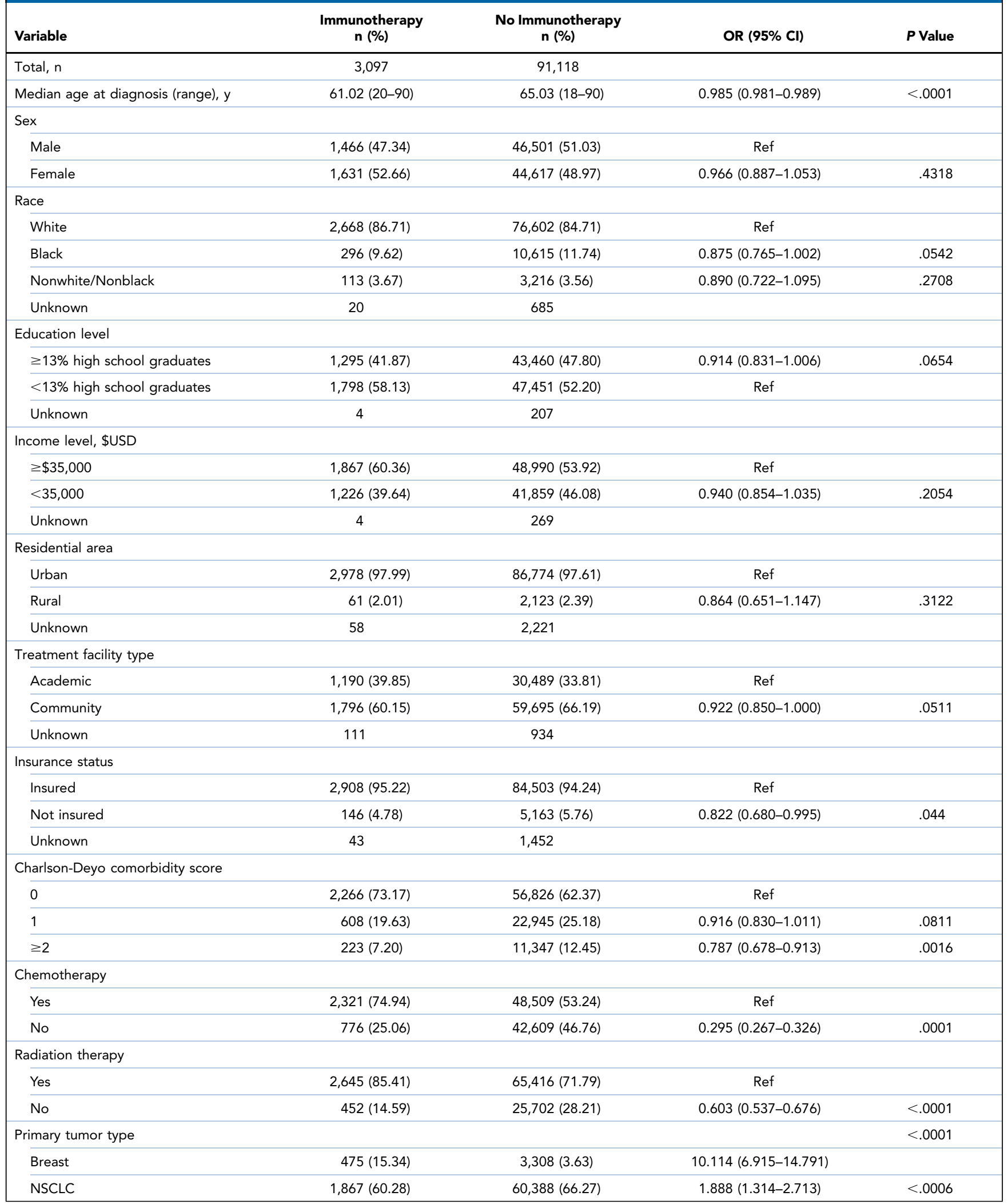




\begin{tabular}{|c|c|c|c|c|}
\hline Variable & $\begin{array}{c}\text { Immunotherapy } \\
\mathbf{n}(\%)\end{array}$ & $\begin{array}{c}\text { No Immunotherapy } \\
n(\%)\end{array}$ & OR $(95 \% \mathrm{Cl})$ & $P$ Value \\
\hline SCLC & $48(1.55)$ & $15,223(16.71)$ & $0.169(0.106-0.268)$ & $<.0001$ \\
\hline Other lung cancer & $31(1.00)$ & 6,495 (7.13) & $0.608(0.364-1.018)$ & .0583 \\
\hline Melanoma & $596(19.24)$ & $2,695(2.96)$ & $21.706(14.913-31.591)$ & $<.0001$ \\
\hline CRC & $49(1.58)$ & $842(0.92)$ & $4.166(2.591-6.698)$ & $<.0001$ \\
\hline Renal cell carcinoma & $31(1.00)$ & $2,167(2.38)$ & Ref & \\
\hline \multicolumn{5}{|l|}{ Year of diagnosis } \\
\hline 2010-2013 & $909(29.35)$ & $60,449(66.34)$ & $0.187(0.172-0.204)$ & $<.0001$ \\
\hline 2014-2015 & $2,188(70.65)$ & $30,669(33.66)$ & Ref & \\
\hline
\end{tabular}

Abbreviations: CRC, colorectal cancer; NSCLC, non-small cell lung cancer; OR, odds ratio; SCLC, small cell lung cancer.

improved OS (HR, 0.739; 95\% CI, 0.782-0.836; $P<.0001$ ) compared with CRT alone (Table 3).

\section{Discussion}

The objective of this study was to compare survival outcomes between patients with BMs treated using

A

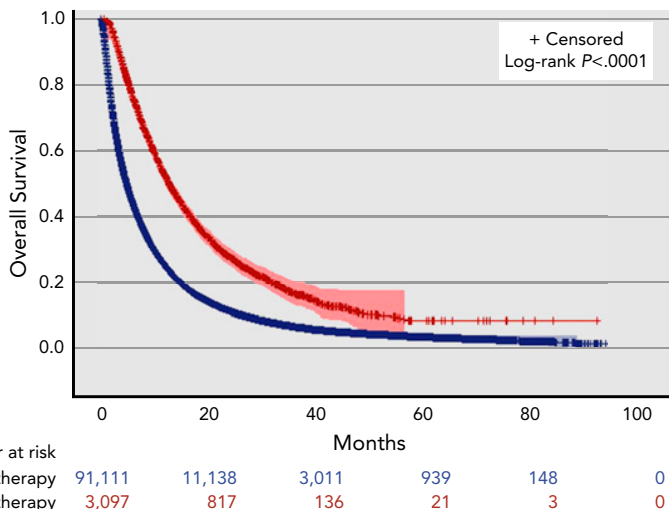

C

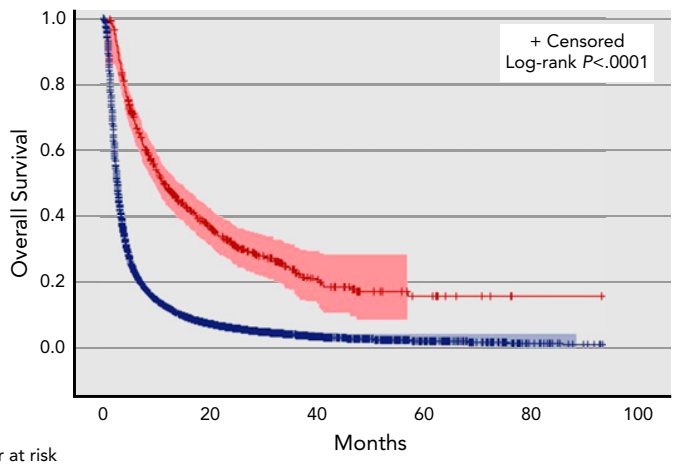

Number at risk

No immunotherapy $24,282 \quad 1,564$

Immunotherapy $\quad 647 \quad 178$ immunotherapy combined with current standard-ofcare treatment regimens and those who received only standard-of-care treatment regimens. Our analysis demonstrates a significant OS benefit for patients treated using immunotherapy combined with other treatments in both univariable and multivariable analyses.

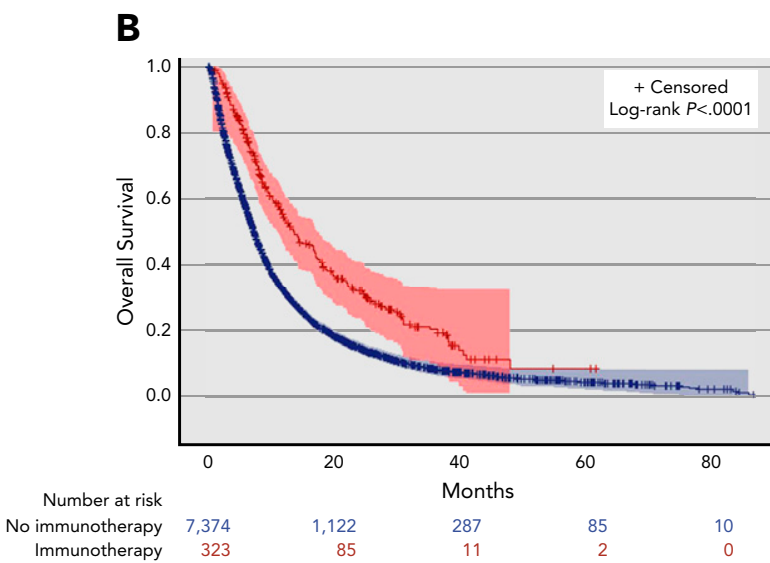

D

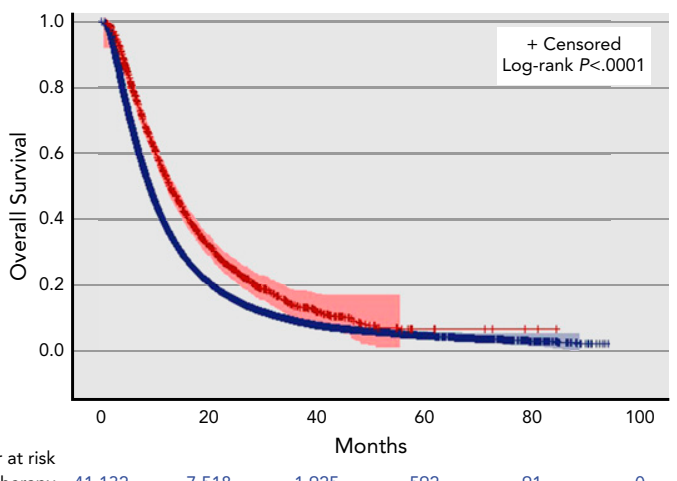

Number at risk

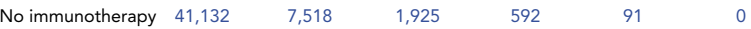
$\begin{array}{llllll}\text { Immunotherapy } & 1,998 & 516 & 78 & 7 & 2\end{array}$

Figure 1. Overall survival with (red) and without (blue) immunotherapy for (A) all patients and patients who received (B) chemotherapy, (C) radiation therapy, and (D) chemoradiation. 
Table 2. Cox Proportional Hazards Regression Analyses of Factors Associated With Overall Survival

Univariable Analysis

\section{Variable}

Age at diagnosis (continuous)

Sex

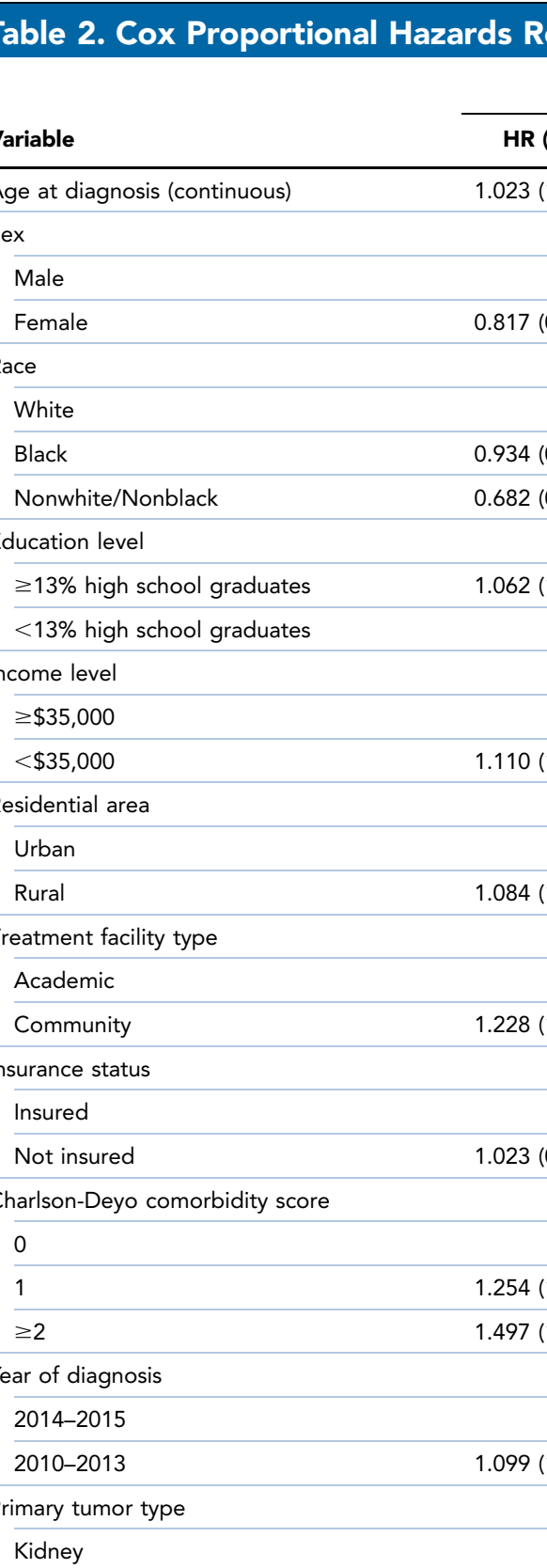

Race

White

Black

Nonwhite/Nonblack

Education level

$\geq 13 \%$ high school graduates

$<13 \%$ high school graduates

Income level

$\geq \$ 35,000$

$<\$ 35,000$

Residential area

Urban

Rural

Treatment facility type

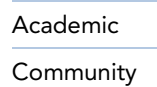

Community

Insurance status

Insured

Not insured

Charlson-Deyo comorbidity score

$$
0
$$

1

$\geq 2$

Year of diagnosis

$$
\text { 2014-2015 }
$$

2010-2013

Primary tumor type

Kidney

Breast

NSCLC

SCLC

Other types of lung cancer

CRC

Melanoma

Chemotherapy

Yes

No

\section{HR $(95 \% \mathrm{Cl})$}

Ref

$.817(0.806-0.828)$

Ref

$0.934(0.914-0.954)$

$<.0001$

.062 (1.048-1.077)

Ref

$<.0001$

Ref

110 (1.095-1.125)

Ref

$.084(1.037-1.133)$

Ref

$.228(1.211-1.246)$

Ref

.023 (0.993-1.053)

Ref

1.254 (1.234-1.274)

$.497(1.466-1.529)$

Ref

1.099 (1.083-1.115)

Ref

0.594 (0.561-0.629)

0.875 (0.837-0.916)

0.947 (0.904-0.993)

$1.768(1.680-1.860)$

$0.784(0.740-0.830)$

1.048 (0.966-1.138)

$0.418(0.412-0.424)$

Ref

$<.0001$

.1343

$<.0001$

.0001

$<.0001$

$<.0001$

$<.0001$
Multivariable Analysis

HR $(95 \% \mathrm{Cl}) \quad P$ Value

$1.012(1.012-1.013)<.0001$

Ref

0.859 (0.847-0.872)

$<.0001$

Ref

$0.931(0.911-0.952)$

$<.0001$

$0.733(0.704-0.763)$

$<.0001$ .511 (NS)

Ref

Ref

$1.053(1.038-1.068)$

$<.0001$

Ref

$<.0004$

.116 (NS)

Ref

$<.0001 \quad 1.170(1.153-1.188)$

$<.0001$

Ref

1.114 (1.080-1.149)

$<.0001$

Ref

1.145 (1.126-1.164)

$<.0001$

$1.221(1.195-1.248)$

$<.0001$

Ref

1.066 (1.050-1.082)

.0001

Ref

$0.708(0.666-0.752)$

$<.0001$

1.047 (0.999-1.097)

.053

$1.225(1.166-1.286)$

$<.0001$

1.348 (1.279-1.422)

$<.0001$

$0.727(0.684-0.773)$

$<.0001$

$1.121(1.030-1.221)$

.0086

$0.455(0.447-0.462)$

$<.0001$

Ref 


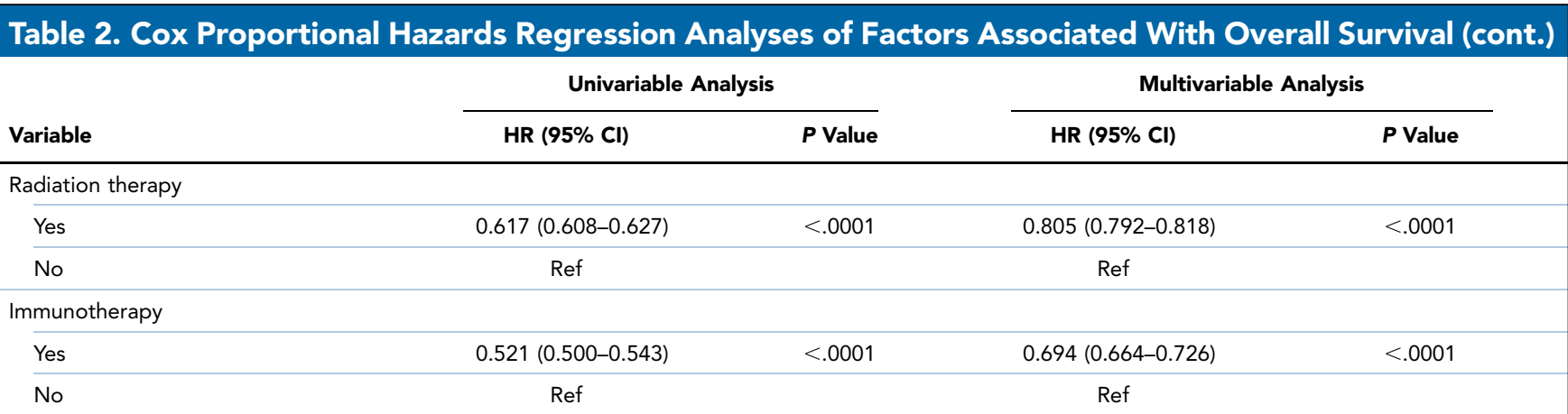

All variables from the univariable analysis with a $P$ value $<.20$ were included in the multivariable analysis. However, for a variable to stay in the final model, its $P$ value had to be $<.10$. The variables for residential area and education both were $P>.10$, and therefore were eliminated from the final model.

Abbreviations: CRC, colorectal cancer; HR, hazard ratio; NSCLC, non-small cell lung cancer; SCLC, small cell lung cancer.

The multivariable analysis was adjusted for age at diagnosis, sex, education level, income level, residential area, treatment facility type, Charlson-Deyo comorbidity score, year of diagnosis, primary tumor type, and receipt of chemotherapy or RT. Receipt of immunotherapy, chemotherapy, and RT; female sex; black and nonwhite/ nonblack race; treatment in an academic facility; Charlson-Deyo comorbidity score of 0 ; and younger age were positive prognostic factors for OS.

Patients receiving immunotherapy + chemotherapy, immunotherapy + RT, or immunotherapy + CRT had longer median survival time than their counterparts. The median survival time noted in our study is similar to that in other published studies. ${ }^{30-32}$ Importantly, our data suggest that immunotherapy improves OS in patients with cancer by approximately 5.5 months, regardless of the standard cancer treatment modalities they receive, such as chemotherapy, RT, or CRT.

Previous studies have investigated the impact of immunotherapy combined with either chemotherapy or RT on patients with melanoma. ${ }^{28,31-40}$ Patients who received chemotherapy in combination with immunotherapy had significantly longer OS (11.2 vs 9.1 months) and a better 1 -year survival rate $(47.3 \%$ vs $36.3 \%)$ than those who received chemotherapy alone. ${ }^{37}$ SRS combined with immunotherapy improved OS by 15.5 months (19.9 vs 4.4 months) in one study, ${ }^{32}$ by 16.4 months (21.3 vs 4.9 months) in another, ${ }^{20}$ and by 21.5 months (28.3 vs 6.8 months) in yet another. ${ }^{38}$ Another study reported a significant reduction in distant intracranial failure (HR, 0.48; 95\% CI, 0.29-0.80; $P<.003)$ in SRS + immunotherapy compared with SRS alone. ${ }^{39}$ Furthermore, a study by Tawbi et $\mathrm{al}^{35}$ showed that $57 \%$ of patients with BMs from melanoma achieved an intracranial clinical benefit, with $56 \%$ achieving complete or partial response after receiving nivolumab combined with ipilimumab. In our study, we found that chemotherapy + immunotherapy significantly improved OS, with an HR of 0.72 (95\% CI, 0.68-0.77; $P<.0001)$ compared with chemotherapy without immunotherapy. RT + immunotherapy also significantly improved OS, with an HR of 0.58 (95\% CI, 0.64-0.61; $P<.0001)$ compared with RT without immunotherapy. Our findings are consistent with

Table 3. Univariable and Multivariable Analyses of Combining Immunotherapy With Chemotherapy and RT

\begin{tabular}{|c|c|c|c|c|c|}
\hline \multirow[b]{2}{*}{ Variable } & \multirow[b]{2}{*}{ n (\%) } & \multicolumn{2}{|c|}{ Univariable Analysis } & \multicolumn{2}{|c|}{ Multivariable Analysis } \\
\hline & & HR (95\% Cl) & $P$ Value & HR $(95 \% \mathrm{Cl})$ & $P$ Value \\
\hline \multicolumn{6}{|c|}{ Chemotherapy and immunotherapy combination } \\
\hline Chemotherapy only & $7,375(95.80)$ & Ref & & Ref & $<.0001$ \\
\hline Chemotherapy + immunotherapy & $323(4.20)$ & $0.580(0.507-0.664)$ & $<.0001$ & $0.643(0.560-0.738)$ & \\
\hline \multicolumn{6}{|l|}{ RT and immunotherapy combination } \\
\hline RT only & $24,282(97.40)$ & Ref & & Ref & \\
\hline RT + immunotherapy & $647(2.60)$ & $0.361(0.328-0.396)$ & $<.0001$ & $0.389(0.352-0.429)$ & .0001 \\
\hline \multicolumn{6}{|l|}{ CRT and immunotherapy combination } \\
\hline CRT only & $41,134(95.37)$ & Ref & & Ref & \\
\hline CRT + immunotherapy & $1,998(4.63)$ & $0.724(0.688-0.762)$ & $<.0001$ & $0.793(0.752-0.836)$ & .0001 \\
\hline
\end{tabular}

The multivariable hazard ratios reported for the combination of immunotherapy with chemotherapy, RT, and chemoradiation are based on separate models because the treatment combinations are mutually exclusive. All 3 models were adjusted for the same factors.

Abbreviations: CRT, chemoradiation; HR, hazard ratio; RT, radiation therapy. 
results of prior studies in patients with melanoma showing that immunotherapy was associated with improved OS. ${ }^{31-33,37-40}$ Most importantly, none of the previous studies included patients with NSCLC, breast cancer, SCLC, or CRC.

The improved OS with the addition of immunotherapy to standard treatments may be additive or synergistic. Chemotherapy and RT cause the release of neoantigens and upregulation of inflammatory cytokines, which promote the presentation of the neoantigens in the tumor microenvironment and thereby increase the immunogenicity of the tumor cells, making them better targets for immunotherapy ${ }^{41-44}$ The irradiated tumor cells may also release cellular danger-associated molecular patterns and cytokines that enhance the traffic of immune cells, leading to elimination of the tumor cells. ${ }^{45,46}$ For patients with significant extracranial disease, addition of immunotherapy may improve survival via controlling the extracranial disease. However, for those without or with minimal extracranial disease, the effect of immunotherapy on survival will be mediated through the control of BMs, which will be influenced by the drug permeability of the BBB. Whole-brain RT or SRS before immunotherapy may enhance such permeability.

To our knowledge, our study is the first to use the NCDB to conduct big data analytics and to investigate the impact of immunotherapy on the OS of patients with BMs, regardless of the primary tumor. Together with the findings of prior studies that have reported an improved intracranial response rate with immunotherapy in patients with melanoma, the results of our study warrant future clinical trials of immunotherapy combined with chemotherapy or RT or both in patients with cancer who have BMs, who have been excluded from clinical trials because of their worse prognosis and possible intracranial toxicities. For patients with BMs who do not participate in clinical trials, proper management of BMs still requires multidisciplinary input regarding proper integration of surgery, radiation, and systemic therapies. Furthermore, quality of life of patients and long-term toxicity should be carefully weighed when immunotherapy is recommended.

The strength of our study is the large sample size, which allowed us to adjust for important patient and tumor characteristics in the multivariable analysis. However, our research is not without limitations, and those limitations are inherent to the NCDB, including incomplete data and ascertainment bias, lack of data regarding local control and the cause of death, lack of detailed information on the use of multiagent chemotherapy regimens, and lack of information on the type of immunotherapy and whether a single or combined immunotherapy was used. In addition, not analyzing whether RT was administered intracranially, extracranially, or both and not including patients who received surgery at the primary site are other limitations and may cause selection bias. Although we found that immunotherapy is associated with improved OS, it is not clear whether this improvement is due to treating extracranial disease, reducing the incidence of new BMs, or both. Despite these limitations, the NCDB comprises most patients treated in the United States and is the best available resource outside multicenter, randomized trials to investigate the impact of novel treatments such as immunotherapy on the OS of patients with cancer who have BMs.

\section{Conclusions}

To our knowledge, this study is the first and most extensive investigation comparing survival outcomes between standard-of-care treatment regimens with and without immunotherapy in patients with cancer and BMs. The present research found significantly improved OS in patients receiving standard therapies when combined with immunotherapy. These findings warrant further clinical trials investigating the impact of immunotherapy combined with chemotherapy, RT, or both in patients with cancer who have BMs.

Submitted December 1, 2019; accepted for publication February 7, 2020.

Author contributions: Study design: Amin, Baine, Lin. Data analysis: Amin, Meza. Manuscript writing: Amin, Lin. Manuscript review and editing: All authors

Disclosures: The authors have disclosed that they have not received any financial consideration from any person or organization to support the preparation, analysis, results, or discussion of this article.

Correspondence: Chi Lin, MD, PhD, Department of Radiation Oncology, College of Medicine, University of Nebraska Medical Center, 986861 Nebraska Medical Center, Omaha, NE 68198-6861. Email: clin@unmc.edu

\section{References}

1. Nayak L, Lee EQ, Wen PY. Epidemiology of brain metastases. Curr Oncol Rep 2012;14:48-54

2. Farber $\mathrm{SH}$, Tsvankin $\mathrm{V}$, Narloch JL, et al. Embracing rejection: immunologic trends in brain metastasis. Oncoimmunology 2016;5: e1172153.

3. Ostrom QT, Wright $\mathrm{CH}$, Barnholtz-Sloan JS. Chapter 2 - Brain metastases: epidemiology. Handb Clin Neurol 2018;149:27-42.

4. Michl M, Thurmaier J, Schubert-Fritschle G, et al. Brain metastasis in colorectal cancer patients: survival and analysis of prognostic factors. Clin Colorectal Cancer 2015;14:281-290.
5. Fabi A, Felici A, Metro G, et al. Brain metastases from solid tumors: disease outcome according to type of treatment and therapeutic resources of the treating center. J Exp Clin Cancer Res 2011;30:10.

6. Esmaeilzadeh M, Majlesara A, Faridar A, et al. Brain metastasis from gastrointestinal cancers: a systematic review. Int J Clin Pract 2014;68:890-899.

7. Cohen JV, Kluger HM. Systemic immunotherapy for the treatment of brain metastases. Front Oncol 2016;6:49

8. Kocher M, Soffietti R, Abacioglu U, et al. Adjuvant whole-brain radiotherapy versus observation after radiosurgery or surgical resection of one 
to three cerebral metastases: results of the EORTC 22952-26001 study. J Clin Oncol 2011;29:134-141.

9. Lin X, DeAngelis LM. Treatment of brain metastases. J Clin Oncol 2015; 33:3475-3484.

10. Owyong M, Hosseini-Nassab N, Efe G, et al. Cancer immunotherapy getting brainy: visualizing the distinctive CNS metastatic niche to illuminate therapeutic resistance. Drug Resist Updat 2017;33-35:23-35.

11. Lauko A, Thapa B, Venur VA, et al. Management of brain metastases in the new era of checkpoint inhibition. Curr Neurol Neurosci Rep 2018; 18:70.

12. Ahluwalia MS, Vogelbaum MV, Chao ST, et al. Brain metastasis and treatment. F1000Prime Rep 2014;6:114

13. Puhalla $S$, Elmquist $W$, Freyer $D$, et al. Unsanctifying the sanctuary: challenges and opportunities with brain metastases. Neuro Oncol 2015; 17:639-651.

14. Brown PD, Ballman KV, Cerhan JH, et al. Postoperative stereotactic radiosurgery compared with whole brain radiotherapy for resected metastatic brain disease (NCCTG N107C/CEC.3): a multicentre, randomised, controlled, phase 3 trial. Lancet Oncol 2017;18:1049-1060.

15. Wu YL, Zhou $C$, Cheng $Y$, et al. Erlotinib as second-line treatment in patients with advanced non-small-cell lung cancer and asymptomatic brain metastases: a phase II study (CTONG-0803). Ann Oncol 2013;24: 993-999.

16. Long GV, Trefzer U, Davies MA, et al. Dabrafenib in patients with Val600Glu or Val600Lys BRAF-mutant melanoma metastatic to the brain (BREAK-MB): a multicentre, open-label, phase 2 trial. Lancet Oncol 2012; 13:1087-1095.

17. Yap YS, Cornelio GH, Devi BC, et al. Brain metastases in Asian HER2positive breast cancer patients: anti-HER2 treatments and their impact on survival. Br J Cancer 2012;107:1075-1082.

18. Krop IE, Lin NU, Blackwell K, et al. Trastuzumab emtansine (T-DM1) versus lapatinib plus capecitabine in patients with HER2-positive metastatic breast cancer and central nervous system metastases: a retrospective, exploratory analysis in EMILIA. Ann Oncol 2015;26:113-119.

19. Fan $Y$, Huang $Z$, Fang $L$, et al. Chemotherapy and EGFR tyrosine kinase inhibitors for treatment of brain metastases from non-small-cell lung cancer: survival analysis in 210 patients. Onco Targets Ther 2013;6: 1789-1803.

20. Knisely JP, Yu JB, Flanigan J, et al. Radiosurgery for melanoma brain metastases in the ipilimumab era and the possibility of longer survival. J Neurosurg 2012;117:227-233.

21. Cochran DC, Chan MD, Aklilu M, et al. The effect of targeted agents on outcomes in patients with brain metastases from renal cell carcinoma treated with Gamma knife surgery. J Neurosurg 2012;116:978-983.

22. Di Giacomo AM, Margolin K. Immune checkpoint blockade in patients with melanoma metastatic to the brain. Semin Oncol 2015;42:459-465.

23. Berghoff AS, Fuchs E, Ricken G, et al. Density of tumor-infiltrating lymphocytes correlates with extent of brain edema and overall survival time in patients with brain metastases. Oncoimmunology 2015;5 e1057388.

24. Berghoff AS, Ricken G, Widhalm G, et al. Tumour-infiltrating lymphocytes and expression of programmed death ligand 1 (PD-L1) in melanoma brain metastases. Histopathology 2015;66:289-299.

25. Westphal D, Glitza Oliva IC, Niessner H. Molecular insights into melanoma brain metastases. Cancer 2017;123:2163-2175.

26. Ahluwalia MS, Winkler F. Targeted and immunotherapeutic approaches in brain metastases. Am Soc Clin Oncol Educ Book 2015;35:67-74.
27. Kiess AP, Wolchok JD, Barker CA, et al. Stereotactic radiosurgery for melanoma brain metastases in patients receiving ipilimumab: safety profile and efficacy of combined treatment. Int J Radiat Oncol Biol Phys 2015;92:368-375.

28. Skrepnik T, Sundararajan $\mathrm{S}$, Cui $\mathrm{H}$, et al. Improved time to disease progression in the brain in patients with melanoma brain metastases treated with concurrent delivery of radiosurgery and ipilimumab. Oncoimmunology 2017;6:e1283461.

29. Qian JM, Yu JB, Kluger HM, et al. Timing and type of immune checkpoint therapy affect the early radiographic response of melanoma brain metastases to stereotactic radiosurgery. Cancer 2016;122:3051-3058.

30. Diao K, Bian SX, Routman DM, et al. Stereotactic radiosurgery and ipilimumab for patients with melanoma brain metastases: clinical outcomes and toxicity. J Neurooncol 2018;139:421-429.

31. Tazi K, Hathaway A, Chiuzan C, et al. Survival of melanoma patients with brain metastases treated with ipilimumab and stereotactic radiosurgery. Cancer Med 2015;4:1-6.

32. Silk AW, Bassetti MF, West BT, et al. Ipilimumab and radiation therapy for melanoma brain metastases. Cancer Med 2013;2:899-906.

33. Di Giacomo AM, Ascierto PA, Pilla L, et al. Ipilimumab and fotemustine in patients with advanced melanoma (NIBIT-M1): an open-label, single-arm phase 2 trial. Lancet Oncol 2012;13:879-886.

34. Weber JS, Amin A, Minor D, et al. Safety and clinical activity of ipilimumab in melanoma patients with brain metastases: retrospective analysis of data from a phase 2 trial. Melanoma Res 2011;21:530-534

35. Tawbi HA, Forsyth PA, Algazi A, et al. Combined nivolumab and ipilimumab in melanoma metastatic to the brain. N Engl J Med 2018;379: 722-730.

36. Long GV, Atkinson V, Lo S, et al. Combination nivolumab and ipilimumab or nivolumab alone in melanoma brain metastases: a multicentre randomised phase 2 study. Lancet Oncol 2018;19:672-681.

37. Robert $\mathrm{C}$, Thomas $\mathrm{L}$, Bondarenko I, et al. Ipilimumab plus dacarbazine for previously untreated metastatic melanoma. N Engl J Med 2011;364: 2517-2526.

38. Shoukat S, Marcus DM, Rizzo M, et al. Outcome with stereotactic radiosurgery (SRS) and ipilimumab (Ipi) for malignant melanoma brain metastases (mets) [abstract]. J Clin Oncol 2014;32(Suppl):Abstract 9076.

39. Acharya $S$, Mahmood M, Mullen $D$, et al. Distant intracranial failure in melanoma brain metastases treated with stereotactic radiosurgery in the era of immunotherapy and targeted agents. Adv Radiat Oncol 2017;2:572-580.

40. Murphy B, Walker J, Bassale S, et al. Concurrent radiosurgery and immune checkpoint inhibition: improving regional intracranial control for patients with metastatic melanoma. Am J Clin Oncol 2019;42:253-257.

41. Schumacher TN, Schreiber RD. Neoantigens in cancer immunotherapy. Science 2015;348:69-74.

42. Demaria S, Formenti SC. Role of T lymphocytes in tumor response to radiotherapy. Front Oncol 2012;2:95.

43. Haynes NM, van der Most RG, Lake RA, et al. Immunogenic anti-cancer chemotherapy as an emerging concept. Curr Opin Immunol 2008;20: 545-557.

44. Ma $Y$, Conforti $R$, Aymeric $L$, et al. How to improve the immunogenicity of chemotherapy and radiotherapy. Cancer Metastasis Rev 2011;30:71-82.

45. Barker HE, Paget JT, Khan AA, et al. The tumour microenvironment after radiotherapy: mechanisms of resistance and recurrence. Nat Rev Cancer 2015;15:409-425.

46. Vatner RE, Cooper BT, Vanpouille-Box C, et al. Combinations of immunotherapy and radiation in cancer therapy. Front Oncol 2014;4:325.

See JNCCN.org for supplemental online content. 


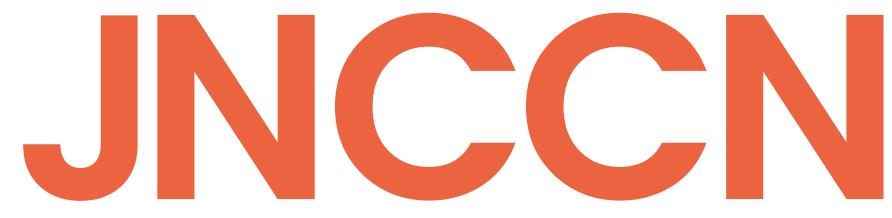

National
Comprehensive
Cancer

Network $^{\oplus}$

\section{JOURNAL OF THE NATIONAL COMPREHENSIVE CANCER NETWORK}

Supplemental online content for:

\section{Impact of Immunotherapy on the Survival of Patients With Cancer and Brain Metastases}

Saber Amin, MD; Michael Baine, MD, PhD; Jane Meza, PhD; and Chi Lin, MD, PhD

J Natl Compr Canc Netw 2020;18(7):832-840

eFigure 1: Overall Survival With and Without Immunotherapy for Patients With Breast Cancer

eFigure 2: Overall Survival With and Without Immunotherapy for Patients With Non-Small Cell Lung Cancer eFigure 3: Overall Survival With and Without Immunotherapy for Patients With Melanoma 
A

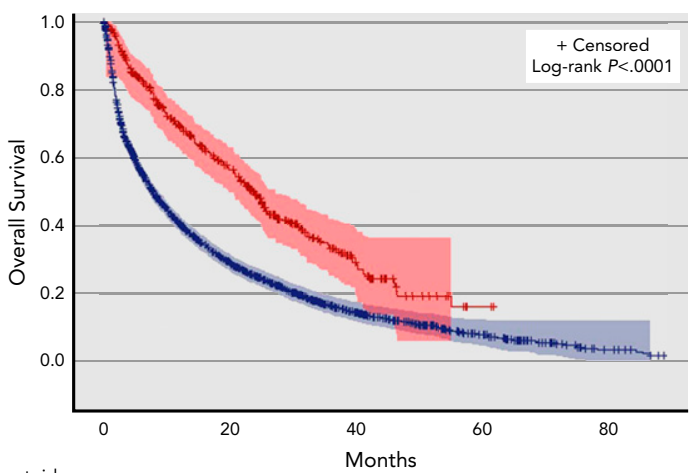

Number at risk

$\begin{array}{rrrrrr}\text { No immunotherapy } & 3,308 & 822 & 298 & 95 & 10 \\ \text { Immunotherapy } & 475 & 201 & 39 & 2 & 0\end{array}$

C

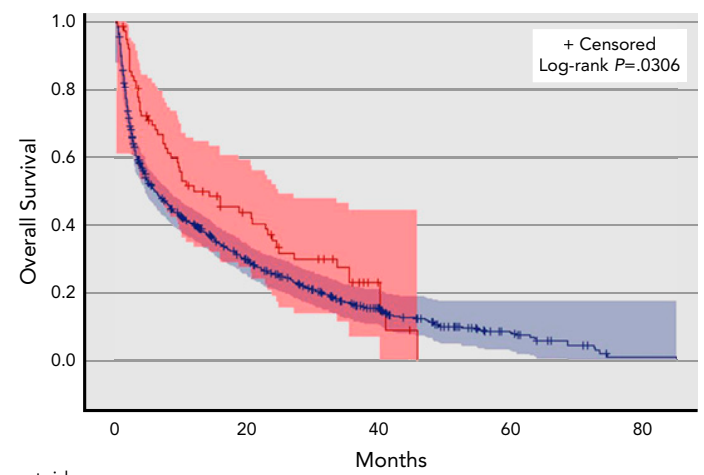

Number at risk

No immunotherapy 922

Immunotherapy 77
B

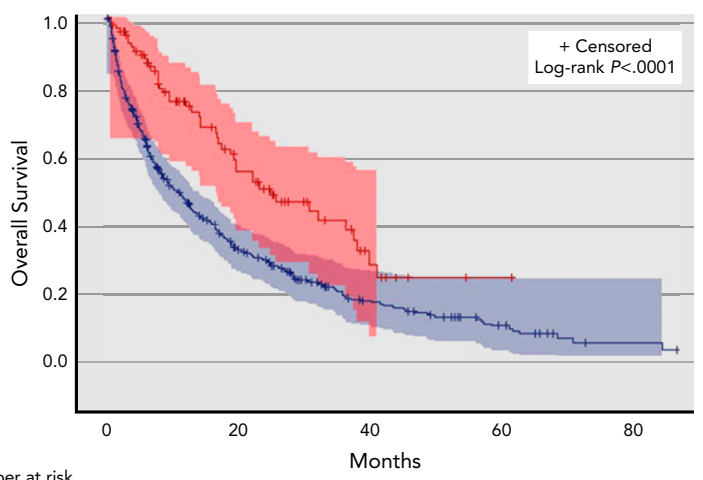

Number at risk

Immunotherapy 95

D

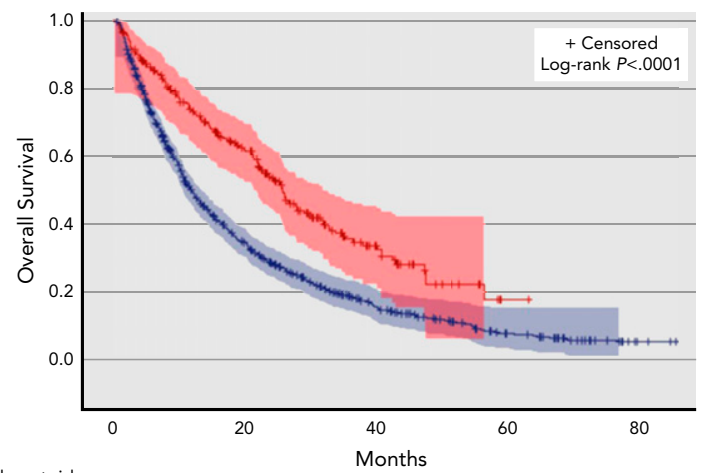

Number at risk

No immunotherapy 1,104

Immunotherapy 269
$122 \quad 40$

$40 \quad 13$

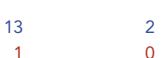

eFigure 1. Overall survival with (red) and without (blue) immunotherapy for (A) all patients with breast cancer and patients with breast cancer who received $(B)$ chemotherapy, $(C)$ radiation therapy, and $(D)$ chemoradiation. 
A

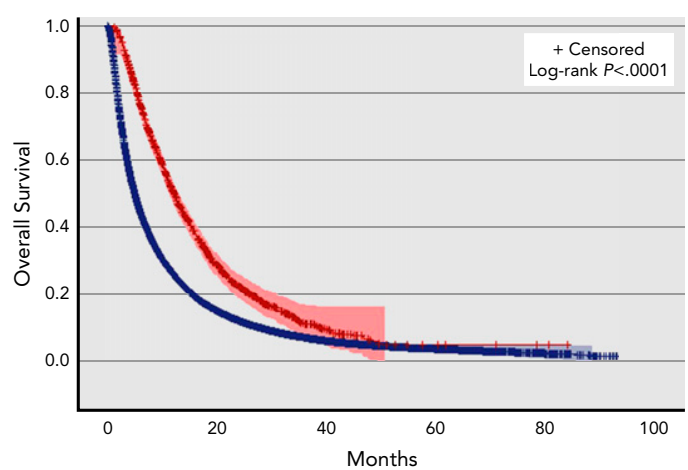

Number at risk

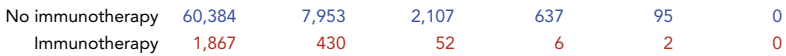

C

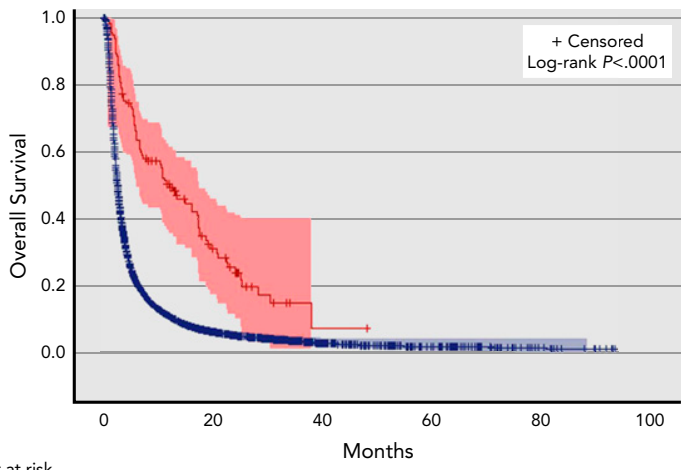

B

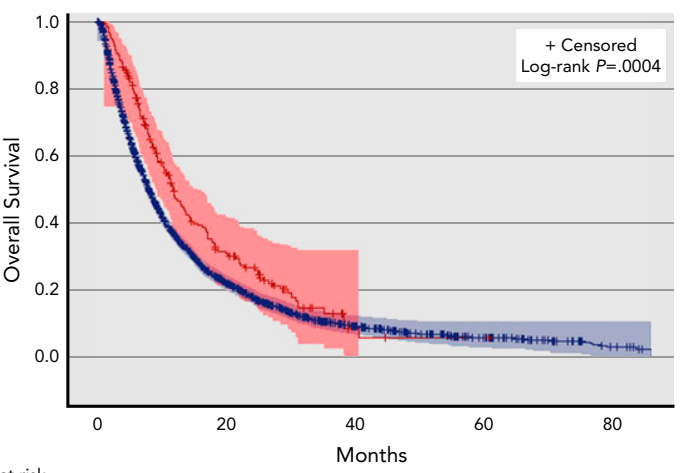

Number at risk

$\begin{array}{lrrrrr}\text { No immunotherapy } & 4,040 & 751 & 193 & 60 & 8\end{array}$

D

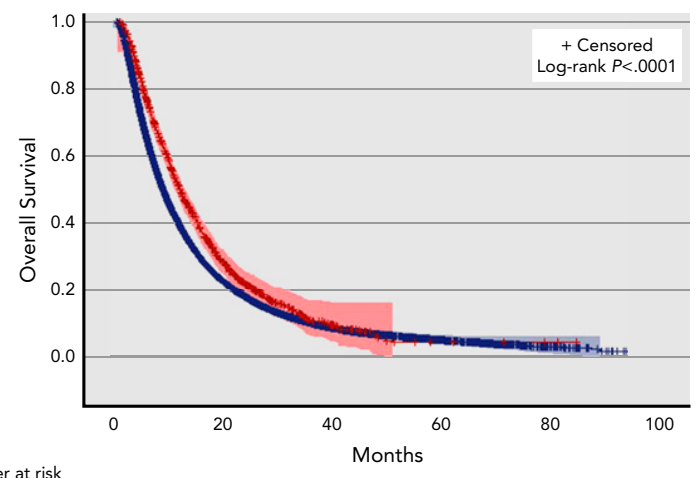

Number at risk

Number at risk

No immunotherapy 17,785

Immunotherapy 114

$\begin{array}{rrr}283 & 90 & 16 \\ 1 & 0 & 0\end{array}$

0 No immunotherapy

$28,658 \quad 5,716$

Immunotherapy $1,544 \quad 356$

1,489
47

eFigure 2. Overall survival with (red) and without (blue) immunotherapy for (A) all patients with non-small cell lung cancer and patients with non-small cell lung cancer who received $(B)$ chemotherapy, $(C)$ radiation therapy, and $(D)$ chemoradiation. 
A

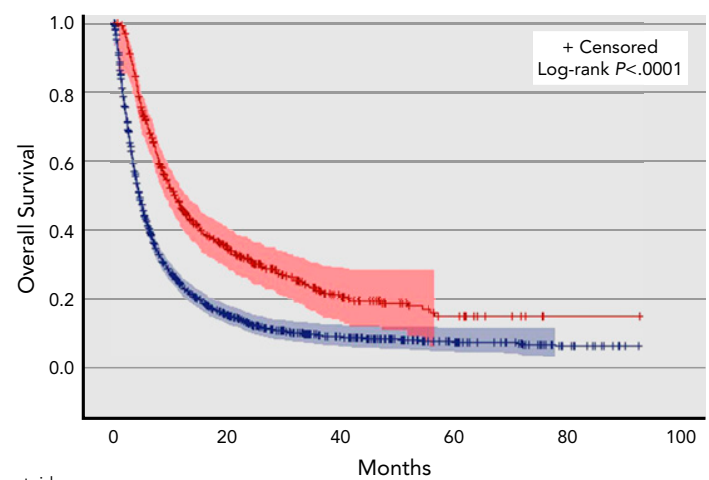

Number at risk No imm No immunotherapy

\section{C}

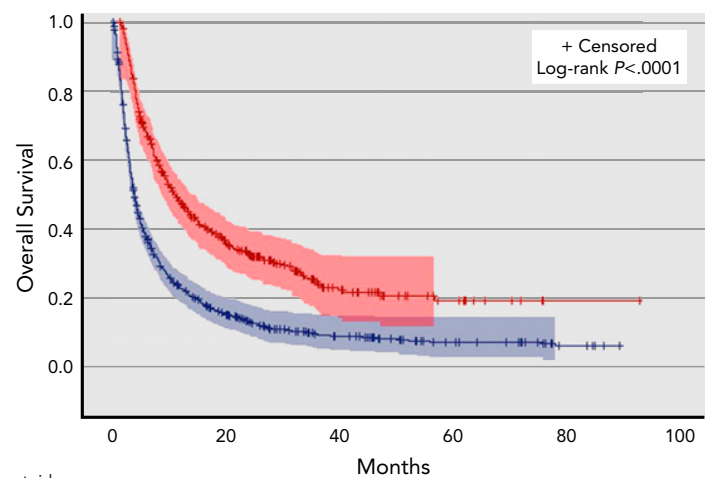

Number at risk

No immunotherapy $\quad 1,054 \quad 139$

Immunotherapy $448 \quad 126$
B

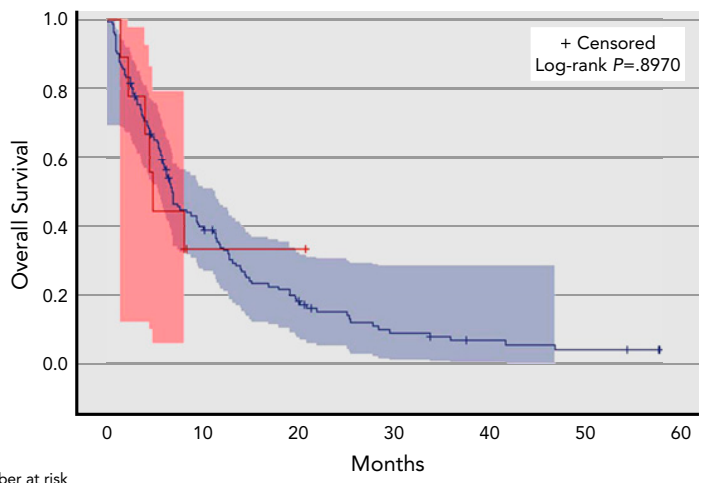
No immunotherapy 130 Immunotherapy

\section{D}

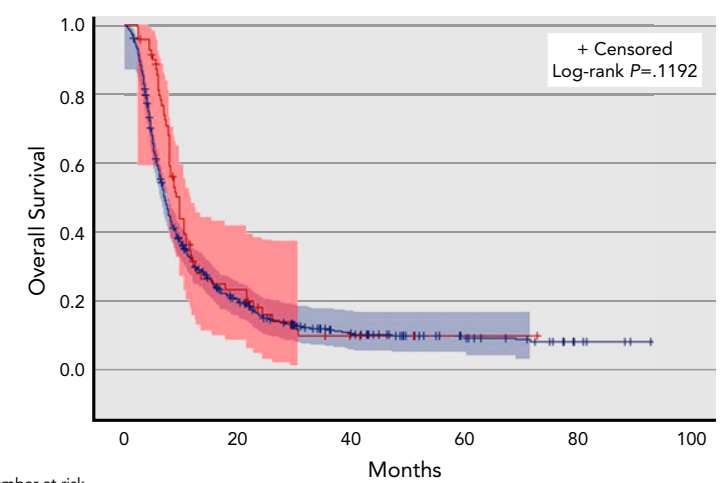

Number at risk

No immunotherapy $768 \quad 138$

Immunotherapy 71

eFigure 3. Overall survival with (red) and without (blue) immunotherapy for (A) all patients with melanoma and patients with melanoma who received $(B)$ chemotherapy, $(C)$ radiation therapy, and $(D)$ chemoradiation. 